\title{
Getting social: Public Health's increasing awareness of the social determinants of health
}

\author{
Timo-Kolja Pförtner • Matthias Richter
}

Published online: 11 January 2011

(C) Springer-Verlag 2011

Even though the media and industry continue to tell us that lifestyle choices are both a threat to and the salvation of our health, there is a growing consensus in Public Health research that health and longevity are substantially determined by nonmedical and non-behavioural factors (Raphael 2006, Richter \& Hurrelmann 2009). Most of today's health problems can be traced back to the social conditions in which we live and work. The report of the WHO Commission on Social Determinants of Health is a new milestone in documenting this shift in perspectives (CSDH 2008). Accordingly, health and health inequalities are increasingly understood as the result of an interdependent process of contextual conditions (such as governance and policy), socioeconomic position, health care systems and life circumstances (Starfield 2007). These social determinants influence a broad set of an individual's resources as well as biological, psychological and behavioural reactions that further shape health. Each of these factors contributes to the explanation of health and illness, and constitutes a complex dialogue between structure and agency that is increasingly being rewritten by Public Health research. The contributions in the current issue of the Journal of Public Health exemplify the increasing awareness of Public Health researchers by focussing on welfare state regimes, social inequalities, psychosocial resources and various aspects of health care.

T.-K. Pförtner

SOCLIFE Research and Training Group, University of Cologne, Cologne, Germany

\section{Richter $(\square)$}

Institute of Social and Preventive Medicine, University of Bern, Niesenweg 6,

3012 Bern, Switzerland

e-mail: mrichter@ispm.unibe.ch
Several papers in this issue deal explicitly with the wider determinants of health: The first article from Hurrelmann et al. takes a broad perspective and introduces an analysis of the impact of different welfare state regimes on population health and health inequalities. The authors present an encompassing overview of the existing social policy and public health literature, and provide a framework that outlines potential pathways linking welfare policy, living conditions and population health. The proposed theoretical approach on the association between welfare state regimes und health facilitates the understanding of health inequalities within and between countries. Using a macro-level approach, Chan analyses the impact of demographic change, economic conditions and health care supply on infant mortality in Singapore. He shows that the level of infant mortally is directly reduced by health care provision, which, in turn, is affected by the level of economic hardship (income per capita and inflation rate) and demographic changes (marriage level, female education level, birth rate). Stroebele et al. take a closer look at contextual conditions of individual health by assessing variety and pricing of selected foods in different socioeconomic districts in Berlin, Germany. Interestingly, when directly comparing the number of grocery stores, the variety of fresh fruits and vegetables being offered, and food prices in low vs. high socioeconomic districts, the authors found no differences that would account for health inequalities. Baiden et al. study the association between satisfaction with housing and the ability to cope with daily demands in Accara, Ghana. Their findings indicate that satisfaction with housing is lower among higher age groups, crowded and compound households, and less educated people. Taking these and other indicators of housing and socioeconomic conditions into account, the ability to cope with daily demands is significantly reduced for people who are dissatisfied with 
their housing conditions. Rostila et al. examine the organisational climate and culture within the Finnish public health and social services, and emphasise the various aspects of contextual differences. Based on a differentiation between working units and upper organisational levels, they conclude that there are different forms of organisational climate and culture within Finnish public human service organisations that have emerged by different mechanisms in these levels.

Two studies focus on more proximate determinants of health, such as individual conditions, resources and behaviour. In their analysis Gao and Chen analyse the association between different aspects of social support and sexually transmitted infections in Canada. After adjustment for health behaviours, socioeconomic conditions and marital status, a consistent negative association between different types of social support and risks of sexually transmitted infections in women aged 15-24 years was found. Although these results could not be replicated for older women and men, the study underlines the importance of social support for sexually transmitted infections in young women. In a second study, Draxler et al. report findings on the relationship between practising Asian martial arts and health-related quality of life in Germany. Based on three samples composed of a reference group and two different samples of people practicing martial arts, the authors find significant differences between martial artists and the general population for all physical and some psychological elements of the SF-36 questionnaire.

The remaining studies in the current issue shed light on different dimensions of health care services. Hasseler et al. present results from patient-oriented interviews on barriers to and resources for effective diabetes care management in Germany. The patients' views on possible coping strategies, healthy lifestyle resources and barriers hampering the adoption of healthy behaviours lead the authors to conclude that support for people with type 2 diabetes in primary care and home settings is needed. The study from Piso et al. presents findings from two DELPHI discussions on the introduction and funding of vaccines. Based on interviews with international immunisation experts they propose a structured, comprehensive and transparent framework for decision-making in vaccine introduction that will strengthen vaccination as an effective public health tool. Chong et al. evaluate the level of patients' and physicians' acceptance of generic medicines and assess the achieved cost savings from substituting these for brand name medicine requests in Malaysia. Their results indicate that generic medicine is highly accepted among physicians and patients, and that a positive substitution contributes significantly to a reduction of expenditures on medicines. Finally, the study from Manova et al. examines the influence of generic and/or therapeutic competition on market share and prices of cardiovascular medicines in Bulgaria. The introduction of generic products for cardiovascular medicines is accompanied by changes in markets and a delayed price reduction. In line with Chong et al., the authors emphasise the implementation of generic substitution policies to control the demand for and dispensing of generic medicines.

Together, these studies provide important additional insight into the social determinants of health and underline the increasing awareness of the social dimension in Public Health. As these studies must be considered a first step in future prospects of Public Health research, further efforts are strongly needed to close the social gap in Public Health.

\section{References}

Commission on Social Determinants of Health (2008) Closing the Gap in a Generation: Health equity through action on the social determinants of health. Final Report of the Commission on Social Determinants of Health. World Health Organization, Geneva

Raphael D (2006) Social Determinants of Health: An Overview of Concepts and Issues. In: Raphael D, Bryant T, Rioux M (eds) Staying Alive. Critical Perspectives on Health, Illness, and Health Care. Canadian Scholars' Press, Toronto, pp 115-138

Richter M, Hurrelmann K (2009) Gesundheitliche Ungleichheit. Grundlagen, Probleme, Perspektiven [Social inequalities in health: principles, problems, perspectives. 2nd Edition]. Wiesbaden: VS Verlag für Sozialwissenschaften

Starfield B (2007) Pathways of influence on equity in health. Soc Sci Med 64(7):1355-1362 